\title{
A pesquisa em leitura e o uso das tecnologias: Translog e EEG
}

\author{
Reading research and the technologies: Translog and EEG ${ }^{1}$ \\ Claudia Finger-Kratochvil \\ Universidade Federal da Fronteira Sul - Chapecó - Santa Catarina - Brasil \\ Luciane Baretta \\ Universidade Estadual do Centro-Oeste - Guarapuava - Paraná - Brasi
}

\begin{abstract}
Resumo: A proposta deste trabalho é discutir o design metodológico e alguns resultados de duas pesquisas conduzidas em nível de doutorado, sobre o processo de compreensão em leitura em língua materna, incorporando a utilização de duas ferramentas tecnológicas: o Translog e o EEG (eletroencefalograma). Considerando as inúmeras possibilidades e a complexidade dos estudos na área de cognição e os altos custos das tecnologias mais avançadas, comumente associadas à área (e.g., fMRI, PET scan, NRCIS), os trabalhos aqui abordados destacam a importância e a viabilidade do uso de metodologias diversas a fim de construir um espectro de compreensão mais acessível às necessidades de pesquisa na área de processamento em leitura.
\end{abstract}

Palavras-chave: Processamento em leitura; Translog; EEG

\begin{abstract}
This paper intends to discuss the methodological design and some of the results from two $\mathrm{PhD}$ research on the reading comprehension process that adopted two technological tools: Translog and EEG (electroencephalogram). Having in mind the diverse possibilities and the complexity of the studies in cognition as well as the high costs of more advanced technologies traditionally used in such investigations, like: fMRI, PET scan, NRCIS, the two studies try to highlight the importance and the viability of other tools, so as to contribute to the needs within the area of reading comprehension research.
\end{abstract}

Keywords: Processing in reading; Translog; EEG

\section{Introdução}

A emergência das ciências cognitivas, na metade do século $\mathrm{XX}$, e os avanços tecnológicos nas últimas décadas possibilitaram o desenvolvimento e o aprimoramento de diferentes pesquisas investigativas acerca dos aspectos cognitivos inerentes à aquisição e ao uso da linguagem. Diferentemente dos estudos comportamentais, com pacientes com e sem lesão cerebral, as pesquisas que se utilizam de ferramentas tecnológicas abrem "janelas de possibilidades" para se investigar, sob diferentes perspectivas, a produção e o processamento da linguagem.

Em se tratando, especificamente, da habilidade da leitura, pesquisas fundamentadas na psicolinguística

\footnotetext{
1 Número de referência University of Auckland Human Participants Ethics Committee: 2007/104.
}

entendem que o ato de ler é resultado da interação entre o texto e o leitor, em um determinado momento. $\mathrm{O}$ interesse em compreender como se efetiva a construção deste tripé que a princípio deverá resultar na compreensão textual é um tema que tem estimulado inúmeras pesquisas e debates teóricos e educacionais, nacional e internacionalmente, como é o caso, por exemplo, da Cátedra da UNESCO, da International Society of Applied Pshycholinguistics (ISAPL), dos congressos da Associação Brasileira de Linguística (ABRALIN), e Linguística Aplicada do Brasil (ALAB), da Jornada Nacional de Alfabetização, da Jornada Neurociências, Leitura e Educação em Destaque, dentre outros eventos.

Motivados e alicerçados em diferentes áreas do conhecimento, como a psicolinguística, a psicologia cognitiva, as neurociências e a análise do texto, pesquisadores têm buscado investigar as diferentes etapas 
envolvidas no processamento da informação, desde o momento em que o leitor visualiza uma palavra na página até a compreensão ser atingida. Resultados dessas pesquisas podem ser encontrados em periódicos temáticos, como The Neuroscience of Reading, organizado por Tomitch, em $2012^{2}$, vinculado à revista Ilha do Desterro, publicada pelo Programa de Pós-Graduação em Inglês da UFSC e disponível online, contemplando artigos de pesquisadores brasileiros e estrangeiros que investigam tanto os processos de nível mais baixo da leitura, como a decodificação e o acesso lexical, quanto de níveis mais elevados como a inferência e o monitoramento da compreensão; a Letrônica, Revista Digital do Programa de Pós-Graduação em Letras da PUCRS, organizada por Buchweitz, Limberger e Kramer, que apresenta, na primeira parte do primeiro número do volume $7 \mathrm{de}$ 2014, pesquisas relacionadas à área da psicolinguística, englobando investigações do processamento e da percepção dos diversos níveis da língua (fonológico, morfológico, sintático e semântico-lexical) e de processos cognitivos complexos (compreensão, produção de resumos, revisão textual e fluência verbal); em livros, como o Reading in the Brain, de Dehaene, traduzido para o português, por Scliar-Cabral, em 2012, com o título: Os neurônios da leitura; e o livro organizado por Wannmacher Pereira e Guaresi: Estudos sobre leitura: Psicolinguística e interfaces, também publicado, em 2012, e disponível online.

As interfaces provenientes de diferentes áreas do conhecimento, como a psicologia cognitiva e a neurociência têm proporcionado novas (e por que não inovadoras?) possibilidades de ferramentas tecnológicas que podem ser utilizadas para melhor compreender o processamento em leitura corroborando, em muitos casos, resultados de estudos experimentais, ou, trazendo novas e diferentes interpretações de dados que, por sua vez, abrem espaço a novos questionamentos teórico-metodológicos e, consequentemente, o desenvolvimento de estudos e a continuidade de pesquisas.

Tendo em vista este pano de fundo, a proposta deste texto é discutir o design metodológico e alguns resultados das pesquisas de doutorado, conduzidas pelas autoras, sobre a compreensão em leitura, incorporando a utilização de duas ferramentas tecnológicas: o Translog e o EEG (eletroencefalograma). Considerando a complexidade dos estudos na área de cognição e os altos custos das tecnologias mais avançadas, os dois trabalhos aqui abordados destacam a importância do uso de metodologias diversas a fim de construir um espectro de compreensão mais acessível às necessidades de pesquisa na área da leitura, mais especificamente, quanto aos aspectos cognitivos envolvidos neste processo.

\section{O Translog}

Conforme mencionamos, uma ferramenta tecnológica que nos possibilita estudar o processamento e a compreensão em leitura, considerando a arquitetura metodológica projetada, é o programa Translog. Apresentaremos e discutiremos um recorte de trabalho de maior amplitude - tese ${ }^{3}$-, envolvendo diferentes instrumentos, considerando seus objetivos, metodologia e tecnologias. Dessa forma, o Translog fez parte de uma pesquisa experimental, com abordagem psicolinguística, nos momentos antes e depois de um Período de Ensino e Aprendizagem - PEA. Para o desenvolvimento do trabalho, buscamos inicialmente conhecer a compreensão leitora dos participantes, estudantes de graduação em seu primeiro ano, por meio de um teste de leitura, formulado com base nos critérios do Programa Internacional de Avaliação de Estudantes (PISA). A partir desses resultados, estabelecemos dois grupos de estudantes a serem acompanhados: um composto por estudantes com alta compreensão e outro com baixa compreensão. Com foco no desenvolvimento de estratégias de aquisição de conhecimento lexical, isto é, estratégias morfológicas, contextuais e de uso do dicionário, propusemos para a leitura, somando ao conjunto do experimento o método/a técnica dos Protocolos Verbais (Think Aloud Protocols - TAPs) registrada em áudio e vídeo, dois textos, com e sem pseudopalavras, antes e depois de um período de ensino e aprendizagem que buscou trabalhar o uso das estratégias mencionadas e sua contribuição no processo de compreensão em tarefas de leitura.

Utilizando-nos do Translog, manipulados os textos para sua apresentação em unidades menores (períodos), nos foi possível acompanhar e registrar a progressão do processo de leitura, isto é, a progressão da leitura das unidades do texto determinada pelo leitor. Esses dados, por sua vez, passaram a compor o conjunto de dados a ser tratado em metodologia de triangulação, visando a compreender o papel do conhecimento lexical e o desenvolvimento de estratégias de aquisição lexical (contextuais, morfológicas e uso de dicionário) e seu papel no processo de compreensão leitora. Nesse recorte, trabalharemos com as medidas de tempo para a realização das tarefas a fim de analisar os dados gerados pela interface do Translog.

\footnotetext{
2 A referência completa das obras mencionadas, no corpo do texto, encontra-se ao final do artigo.

3 FINGER-KRATOCHVIL, C. Estratégias para o desenvolvimento da competência lexical: relações com a compreensão em leitura. Florianópolis, 2010. 677p. (v.) I, (v.) II. Tese de Doutorado: área de Psicolinguística.UFSC.
} 


\subsection{Translog e seus projetos}

O Translog, programa criado por Jakobsen ${ }^{4}$, Schou e equipe, originalmente, para a pesquisa dos processos de escrita em tradução na Copenhagen Business School, tem sido usado para investigações em leitura, pois possui interface que possibilita coletar dados a respeito da realização de tarefas pelos participantes. Enquanto o participante responde às tarefas propostas na tela do computador, o programa registra os movimentos realizados no teclado e mouse e o tempo de duração dessas ações em outra interface. Ao final, se pode rastrear essas ações, por meio de registro codificado off-line e, dessa forma, saber que atividade o participante realizou e quanto tempo ele dedicou para a sua realização.

Para isso, primeiro, é necessário criar um projeto que se torna arquivo de entrada na interface "TranslogSupervisor". O projeto passa a ter unidades e são demarcadas por caracteres, e.g., *; <unit $>$; $<$ dict $>=$. Destas marcações, apenas os asteriscos são vistos pelos participantes ao carregarem os projetos para execução da leitura, tendo por função lembrá-los de pensar em voz alta, nessa proposta de trabalho, na interface "TranslogUser". Assim, ao não saberem da existência das outras marcações, tampouco poderem percebê-las, eles não sofrem sua interferência na realização da tarefa.

Para os propósitos do estudo em discussão, criamos vários projetos ${ }^{5}$, apresentando aos participantes os textos em unidades menores, contendo palavras e pseudopalavras, observadas a densidade lexical e a complexidade sintática desses (textos) a fim de que se assemelhassem, bem como a escolha das palavras e pseudopalavras marcadas, a figurar ou não no dicionário on-line, - outro recurso do programa. Cada um desses projetos compreendia em um texto, nomeado pela palavra-chave, por exemplo, Plantas, Infiéis, Titãs.

O uso de pseudopalavras buscou eliminar o efeito de frequência e/ou possível familiaridade com lemas que

\footnotetext{
4 Site $<$ http://www.translog.dk $>$

5 Diferentes aspectos e critérios foram observados na construção dos projetos, criados e mencionados no texto, mas não serão discutidos no corpo deste trabalho. Recomendamos que o leitor consulte o trabalho original. Eles são importantes para o aprofundamento e melhor compreensão dos resultados dos participantes. Por exemplo, importa entender que os types escolhidos (com fins de registro no cômputo dos dados relativos à consulta no Dicionário on-line), apresentavam índices iguais ou menores que $0,15 \%$ - hapax legomena - de acordo com as faixas de frequência de palavras apresentadas pelo Banco de Português (BERBER SARDINHA, 2005) ao se considerar a possível familiaridade.

6 Esse índice de conhecimento refere-se ao conhecimento semântico que, segundo pesquisas, interfere na leitura e compreensão, mas que, entretanto, parece não impedir a leitura em voz alta em virtude da obediência aos padrões grafêmico-fonológicos (no caso desta pesquisa da língua portuguesa) (SCLIAR-CABRAL, L; 1993; NATION; SNOWLING, 1998, 2004; PERFETTI, 2007; NATION; COCKSEY, 2009).

7 Para mais informações, veja o trabalho na íntegra: FINGERKRATOCHVIL, 2010.

8 "[...] mental effects take time."
}

poderiam ser elencados para preencher, o type, de sentido, pois não existiriam em qualquer texto ou dicionário anteriormente elaborado, apresentando índices de conhecimento próximos a zero ${ }^{6}$ diante de sua configuração (GROFF, 2008; NY; CORDIER, 2004; VENEZKY, 1999) e, de certa forma, aumentando, assim, a necessidade de os participantes recorrerem ao uso de estratégias para a sua compreensão. A criação das pseudopalavras teve por alvo a utilização de afixos e radicais de palavras já existentes na língua com o intuito de provocar os participantes e verificar o seu comportamento diante dessa configuração, de um possível conhecimento parcial e de aplicação de estratégia para resolução do problema ${ }^{7}$.

A interface TranslogSupervisor propiciou, para cada momento da testagem, um arquivo $(* . \log )$ em que se verificou a representação linear dos movimentos dos participantes no software de acordo com as medidas temporais estabelecidas - para este trabalho a unidade de tempo para o registro de pausa foi de .01 segundo, i.e., um asterisco vermelho representa um segundo de pausa. Por intermédio do registro off-line, tivemos acesso a dados que, quando não manifestos nos protocolos verbais (ThinkAloud Protocols - TAPs), método também utilizado, ficariam ocultos para a pesquisadora: e.g., tempo de leitura de determinada unidade textual, consultas ao D-on (Dicionário on-line), anotações feitas. Da mesma forma, os TAPs tornaram disponíveis informações sobre outros comportamentos dos participantes que não poderiam ser materializados pelo registro off-line do Translog ou apenas pela gravação de áudio, e.g., interesse e motivação no decurso da realização da tarefa (RUDDELL, 1994), fases de concentração e distração, entre outros (ALVES, 2001).

\subsection{Os dados e as medidas temporais}

As medidas temporais são característica das pesquisas cognitivas, pois "[...] os eventos mentais tomam tempo"8 (ASHCRAFT, 1994, p. 36, grifo do autor, tradução nossa). Compreendemos, normalmente, o fator tempo, como um indicador da carga de demanda cognitiva imposta ao participante. Quanto mais tempo ele leva para o desenvolvimento de uma atividade, entendemos, por exemplo, que menos automatizados estão os processos cognitivos relativos à tarefa, exigindo, assim, mais tempo para o processamento; ou, ainda, que a complexidade da tarefa pode ser tão grande que impinge ao participante a ativação de vários processos e subprocessos cognitivos, acarretando certa sobrecarga e, portanto, requerendo mais tempo para o processamento. Por essas razões, o tempo empregado, na realização de uma tarefa, não pode ser visto isoladamente e, nem tampouco, como algo positivo ou negativo, mas precisamos observá-lo sob as lentes dos propósitos de cada estudo. 
Considerando, então, os objetivos desta pesquisa e o modo como as estratégias são tratadas, i.e., habilidades sob análise ("skills under consideration"), as medidas de tempo foram importantes para ampliar a compreensão de alguns aspectos da aquisição de conhecimento lexical, e.g., o tempo empregado para a resolução de um problema lexical, o tempo dedicado à consulta ao dicionário, e seus desdobramentos e relações e com a competência leitora. Assim, para este trabalho, analisaremos duas das medidas de tempo possíveis:

a) tempo total para a leitura: o tempo total para a leitura foi computado a partir do carregamento da primeira unidade do texto (título), até a leitura da última unidade, considerando-a encerrada quando o participante realiza novo movimento com o mouse e inicia nova tarefa, i.e., a escritura da síntese;

b) tempo de leitura das unidades de texto com pseudopalavra: ao estudar o tempo empregado para a leitura diante de pseudopalavra; portanto, de um estranhamento e de uma possível quebra no fluxo da leitura, buscou-se avaliar o esforço cognitivo do participante para resolver o problema do desconhecimento do item lexical no pré-teste e no pós-teste.

Neste trabalho, buscamos saber se os participantes se beneficiaram do PEA, passando a utilizar com maior eficiência as estratégias de conhecimento lexical e se há correlações entre o seu tempo de leitura nos textos Infiéis e Plantas (ambos com pseudopalavras). Em princípio, espera-se que as estratégias facilitem o processamento e reduzam, assim, o tempo empregado para os processos de solução de problemas de conhecimento lexical - em especial nos períodos com pseudopalavras.

A partir do cômputo do tempo, partimos para algumas análises estatísticas, utilizando o SPSS. Empregamos os testes estatísticos não-paramétricos de Wilcoxon, o rho de Spearman e o $r$ de Pearson para análise das condições e possíveis correlações. A estatística descritiva será relatada no início da discussão de cada teste empregado e seus resultados.

A fim de verificar a existência de correlações para a variável tempo de leitura, em Infiéis e Plantas, antes e depois do PEA, utilizou-se o teste estatístico de Spearman. As medianas das variáveis evidenciam que os participantes demoraram mais tempo na leitura do texto Plantas $(\mathrm{M}=751,00, \mathrm{DP}=620,137)$ (após o PEA) do que na leitura de Infiéis $(\mathrm{M}=566,00, \mathrm{DP}=331,166)$ (antes do PEA). A priori, considerando que os textos e as unidades de leitura tinham extensões diferentes entre si, tendo Infiéis um total de 308 tokens $^{9}$ e Plantas, $543-$

\footnotetext{
9 É importante lembrar que os tokens são entendidos como o número de palavras corridas que compõem o texto.
}

assim, Plantas era $76 \%$ maior em tokens do que Infiéis -, a diferença no tempo de leitura era esperada. Observando os coeficientes de correlação e os valores $p$, verificamos correlação entre o tempo de leitura dos dois textos correlação forte positiva e significativa $(r h o=0,764$, $p=0,006$ ), indicando que os participantes que dedicaram mais tempo para ler Infiéis também empregaram mais tempo para ler Plantas.

Lembramos que, em Infiéis e Plantas, havia unidades de texto que continham pseudopalavras, outras não. A fim de conhecer como os participantes se comportaram em relação à composição das unidades (com ou sem; com 1 ou com 2), antes e depois do PEA, e o tempo de leitura empregado, calculamos, além do tempo médio de leitura total, o tempo médio de leitura para cada tipo de unidade - com e sem pseudopalavra. Baseados nesses dados e utilizando o SPSS, serão discutidos os resultados das correlações par a par, com base no teste estatístico $r$ de Pearson.

A partir da análise da estatística descritiva, verificamos que, em Infiéis, a mediana relacionada ao tempo dedicado pelos participantes para a leitura de unidade sem pseudopalavras $(M=14,76200, D P=9,472187)$ é menor do que a mediana para a leitura de unidade com pseudopalavras $(\mathrm{M}=21,78400, \mathrm{DP}=16,597254)$. Esses resultados evidenciam que os participantes empregaram mais tempo na leitura das unidades com pseudopalavras - UCPsd - do que das unidades sem pseudopalavras - USPsd -, embora as UCPsd, em número de tokens (94 itens lexicais: 30,51\%), fossem, em torno de 50\%, menores do que as USPsd (214 itens lexicais: 69,48\%). O número de tokens, caso a especificidade das unidades não fosse considerada, induziria a pensar que a leitura das UCPsd ocuparia um terço do tempo, enquanto a das USPsd tomaria dois terços, proporcionalmente; contudo, percebemos quase uma inversão dessas proporções de tempo, apesar do número de tokens, pois as UCPsd consumiram $60,38 \%$ do tempo médio empregado em Infiéis.

A mediana da variável tempo de leitura em Plantas para as USPsd $(\mathrm{M}=22,48900, \mathrm{DP}=23880,034)$ também é menor do que a mediana das UCPsd ( $M=47,87200$, $\mathrm{DP}=28564,582)$, embora a relação em número de tokens (262 itens lexicais: 47,89\%) seja quase igual entre as duas variáveis: UCPsd - 262 itens lexicais -, USPsd - 267 itens lexicais -, demonstrando que, mais uma vez, os participantes demoraram mais tempo na leitura das UCPsd. A análise geral dos participantes nos mostra que, com exceção de um participante, todos os demais dedicaram mais tempo para a leitura das UCPsd, lembrando que o número de tokens entre as duas condições era praticamente igual - com uma diferença de apenas cinco entre as duas. 
Embora se verifique em Plantas um maior consumo de tempo para a leitura das UCPsd $(63,85 \%)$ do que para as USPsd (36,15\%), proporcionalmente, os participantes parecem ter reduzido o tempo de leitura, pois o número de palavras para as UCPsd, nesse texto, é maior do que o número de palavras em Infiéis, enquanto a proporção do tempo de leitura é quase o mesmo, $60 \%$. Além disso, outras duas medidas reforçam a ideia do ganho de tempo. Ao comparar a densidade lexical e a complexidade sintática das UCPsd, em ambos os textos, constata-se que a primeira - densidade lexical - é menor em Infiéis (52.12\%) do que em Plantas (56,48\%), do mesmo modo que a segunda - complexidade sintática - Infiéis $(2,14)$, Plantas $(2,20)$. Assim, sendo mais denso e complexo, esperávamos um consumo de tempo maior para a leitura de Plantas, mesmo que proporcional, do que para Infiéis e, na realidade, verificamos um consumo quase equiparável, reforçando, dessa forma, a ideia do ganho em tempo de processamento na leitura em Plantas (depois do PEA).

Outras correlações se estabeleceram entre o tempo de leitura das unidades de texto com - UCPsd - e sem pseudopalavras - USPsd -, e muitas delas foram significativas de moderadas a fortes. Observando a variável tempo de leitura das USPsd de Infiéis, nota-se um coeficiente de correlação forte e significativo com a variável UCPsd no mesmo texto $(r=0,927$, $p<0,001)$, beirando uma correlação perfeita, enquanto com as variáveis de Plantas, verifica-se uma correlação moderada $(r=0,709, p=0,015)$ e outras três, seja com uma $(r=0,691, p=0,019)$ ou duas pseudopalavras $(r=0,773, p=0,005)$, ou independentemente $(r=0,773$, $p=0,005)$ dessa categorização, fortes e todas significativas. A outra variável relativa a Infiéis, i.e., tempo de leitura das unidades com uma pseudopalavra, mostrou correlações moderadas e significativas com as variáveis de Plantas sem pseudopalavras $(r=0,664$, $p=0,026)$, com uma pseudopalavra $(r=0,627, p=0,039)$, e independentemente da categorização do número de pseudopalavras nas unidades $(r=0,609, p=0,047)$. E, para duas pseudopalavras $(r=0,582, p=0,060)$, apesar de os coeficientes serem moderados, o valor de $p$ foi 0,006 , i.e., muito próximo, mas levemente acima do valor de probabilidade $(0,05)$, utilizado nesta pesquisa.

Analisando, ainda, as correlações entre as variáveis de Plantas, constatamos que o tempo de leitura das unidades sem pseudopalavras estabeleceu correlações fortes positivas e significativas com as variáveis tempo de leitura das unidades, com uma $(r=0,700, p=0,016)$ ou com duas pseudopalavras $(r=0,773, p=0,005)$, ou independentemente dessa categorização $(r=0,782$, $p=0,004)$, indicando que quem empregou mais tempo na leitura de unidades sem pseudopalavras também gastou mais tempo na leitura de unidades com pseudopalavras.
Encontramos uma correlação positiva moderada entre a leitura de unidades com uma e com duas pseudopalavras $(r=0,600, p=0,051)$, mas o valor de $p$ foi 0,051 , apenas marginalmente significativo. As variáveis com uma $(r=0,873, p<0,001)$ e com duas $(r=0,882, p<0,001)$ pseudopalavras estabelecem correlações positivas fortes com a variável Plantas Tempo de leitura de unidades com pseudopalavras independentemente do número de pseudopalavras, confirmando as correlações estabelecidas anteriormente.

As correlações entre as variáveis de tempo com e sem pseudopalavras apontam para um comportamento regular por parte dos participantes, ou seja, quanto mais tempo eles empregaram para a leitura de um tipo de unidade de texto, e.g., sem pseudopalavras, também empregaram mais tempo para o outro, em ambos os textos. Esses resultados apresentam um comportamento consistente para a maior parte dos participantes. Em outras palavras, eles encontraram menos dificuldade para ler as unidades de texto sem pseudopalavras e, por isso, foram mais rápidos nelas, enquanto que, ao se depararem com problemas de construção do conhecimento lexical, foram mais cautelosos na leitura. Contudo, conforme pontuamos anteriormente, diante da extensão do conjunto de unidades textuais em cada condição e em cada texto, percebemos modificações no comportamento dos participantes, implicando redução do tempo de leitura o que nos parece apontar para ganhos nos tempos de processamento nos testes após o PEA e, dessa forma, sinalizando benefícios ao compreender o possível uso de estratégias de aquisição do conhecimento lexical para a compreensão leitora dos participantes.

Os resultados ora apresentados são interessantes e nos levam a pensar em novas pesquisas com arranjos metodológicos distintos. Entretanto, vale ressaltar que o Translog, embora seja uma ferramenta mais acessível, tanto em termos de custos quanto de mobilidade, oferece a possibilidade de leitura indireta dos processos cognitivos em ação, considerando que o participante responda de acordo com o a instrução dada. Dessa forma, duas questões são importantes: a) lembrar que é a interpretação dos diferentes dados gerados pelo pesquisador que pode vir a lançar luzes que ampliem a compreensão do processo e, portanto, b) ampliar a amostra e reaplicar a pesquisa são fatores que ajudam na confiabilidade dos resultados.

\section{O eletroencefalograma e o processo de compreensão}

Antes de nos atermos ao eletroencefalograma (EEG), é importante esclarecermos que há uma variedade de ferramentas tecnológicas não invasivas disponíveis aos pesquisadores para investigar os correlatos neurais do 
funcionamento cerebral. Essas ferramentas podem ser categorizadas de acordo com o tipo de informação que elas apresentam: as que oferecem informação de alta resolução espacial e aquelas que apresentam informação de alta resolução temporal (OCDE, 2003).

Tanto o PET scan, sigla para Positron Emission Tomography ou, em português, Tomografia por Emissão de Pósitrons, e o fMRI, functional Magnetic Ressonance Imaging ou RNMf, sigla em português para Ressonância Nuclear Magnética Funcional, apresentam alta resolução espacial, i.e., mostram detalhes milimétricos das áreas cerebrais sendo investigadas. Ambas as tecnologias se utilizam de radioisótopos para monitorar o aumento do fluxo sanguíneo, da glicose e oxigenação das áreas cerebrais, concluindo que estas são as áreas envolvidas na execução de uma dada tarefa, como por exemplo, a leitura (TOMITCH; JUST; NEWMAN, 2004). A principal diferença entre essas duas tecnologias é a necessidade de uma injeção de glicose no paciente para a realização do PET scan. A glicose é ligada a um elemento radioativo (normalmente flúor radioativo, ou Oxigênio-15 para o PET do cérebro), para que as regiões que a estão metabolizando em excesso fiquem em vermelho na imagem criada pelo computador. No fMRI, não há necessidade de tal procedimento invasivo, pois a ferramenta utiliza-se de um forte campo magnético e ondas de rádio para criar imagens detalhadas do fluxo sanguíneo cerebral para detectar as áreas ativas durante o escaneamento (LAGE, 2013; OCDE, 2003; TANNER, 2007).

O EEG, do inglês electroencephalography, diferentemente do PET e do fMRI, apresenta alta resolução temporal ${ }^{10}$ por meio do registro das correntes elétricas desenvolvidas no encéfalo; por esta razão, é possível detectar o momento preciso de uma alteração nas ondas elétricas cerebrais produzidas pelo participante. Sabendose que a transmissão das informações no cérebro ocorre através da corrente de íons, o EEG grava essa atividade elétrica, que é detectada através da utilização de eletrodos (KUTAS; VAN PETTEN, 1994). Tradicionalmente, o EEG é associado a estudos clínicos, como diagnóstico de epilepsia e distúrbios do sono, mas os avanços tecnológicos forneceram aos pesquisadores a possibilidade de mensurar a atividade cerebral associada ao funcionamento sensorial e cognitivo, através do registro de uma atividade cerebral diferente e mais sutil, o ERP (do inglês event-related brain potential).

Basicamente, este aspecto do registro da atividade cerebral é sincronizado a um estímulo ou evento externo, como por exemplo, ler uma palavra, ouvir uma melodia ou visualizar uma imagem, fazendo do EEG uma ferramenta

\footnotetext{
${ }^{10}$ Outra ferramenta que também é conhecida e utilizada em pesquisas por apresentar alta resolução temporal é a magnoeletroencefalografia (MEG).
}

não invasiva, capaz de registrar processos cognitivos como a compreensão em leitura (BROWN; HAGGORT, 2000; KUTAS; SCHMITT, 2003). Para o registro de ERPs, pesquisadores colocam eletrodos, em posições pré-definidas, sobre o couro cabeludo do paciente. Os sinais de ERPs são mensurados em relação a um eletrodo de referência, que é colocado no lobo da orelha, no queixo ou na área central do escalpo do participante; essa área é conhecida como $\mathrm{Cz}$ de acordo com o Sistema Internacional 10-20 (vide LAGE, 2013, p. 165 e 167). A comparação dos sinais elétricos entre o eletrodo de referência e os outros permite aos pesquisadores mensurar a diferença na atividade cerebral através das diferentes áreas do escalpo. Para se obter uma boa amostra dos ERPs em experimentos cognitivos, o número de tarefas para cada tipo de estímulo sendo invvestigado varia de 30 a 50 rodadas.

De acordo com Kutas e Schmitt (2003), as ondas dos ERPs são comumente interpretadas e representadas de acordo com 3 parâmetros: (1) a amplitude, calculada em microvolts e refererenciada a uma base de referência; (2) a latência, que é o tempo a partir do início do estímulo e (3) a polaridade, com o P para a onda positiva e N para a negativa. As flutuações positivas e negativas que podem ser visualizadas em cada ERP são definidas como "componentes" e são nomeadas em função de sua polaridade e latência, em milisegundos. Por exemplo, o N400 representa uma mudança negativa na amplitude, que ocorre a aproximadamente 400 milisegundos após o início do estímulo de interesse. Este componente, que é o principal investigado na pesquisa que iremos relatar, a seguir, é provavelmente um dos mais investigados desde a publicação do artigo seminal de Kutas e Hillyard, em 1980, sobre o processamento semântico de sentenças escritas. Como já mencionado, o N400 é uma onda negativa, com seu ápice ocorrendo por volta dos 400 milisegundos e é associado com a dificuldade dos leitores em integrar um elemento lexical ao contexto anterior. O N1 ou N100, outro componente investigado na pesquisa a ser relatada, é originado por estímulos auditivos e visuais e reflete processos sensoriais precoces e processos relativos à atenção, associados à habilidade do participante de processar o canal de informação (RANEY, 1993).

A partir da breve contextualização sobre EEG e ERPs (para maior aprofundamento, consultar KUTAS; SCHMITT, 2003; LAGE, 2013), passamos ao relato da pesquisa de doutoramento da segunda autora, realizada em parceria entre a Universidade Federal de Santa Catarina (Programa de Pós-Graduação em Inglês) e a Universidade de Auckland, na Nova Zelândia (Centro de Neurociência Cognitiva). O principal objetivo da pesquisa foi a investigação do processo de geração de inferências na compreensão leitora utilizando-se do EEG para coleta 
dos dados. Diferentemente da maioria das pesquisas relativas ao processamento linguístico, esta pesquisa utilizou o EEG para a coleta de dados com excertos mais longos do que palavras ou sentenças isoladas, conforme estudo conduzido por St. George, em 1995. Fizeram parte do estudo 14 estudantes universitários ${ }^{11}$, destros, homens, entre 18 e 31 anos, falantes nativos do inglês. A opção por se trabalhar com homens, somente, deve-se ao fato de eles serem mais lateralizados para a linguagem. Outro fator é o ciclo hormonal feminino que afeta a transferência de informações no cérebro (HAUSMANN, BECKER; GATHER; GÜNTÜRKÜN, 2002). O EEG foi coletado continuamente através de um equipamento com 128 eletrodos, da Eletrical Geodesics Incorporation. 13 sítios/ eletrodos foram selecionados para registrar as ondas cerebrais, de acordo com as regiões de interesse para o estudo, baseando-se em pesquisas anteriores (COULSON; VAN PETTEN, 2002; St. GEORGE, 1995). A coleta de dados foi feita individualmente com cada participante em uma única sessão, de aproximadamente 90 minutos. Após explicação e orientações sobre o experimento, assinatura do $\mathrm{TCLE}^{12}$ e a colocação da touca com os eletrodos, todos os participantes sentaram-se diante da tela de um computador e leram 30 parágrafos narrativos e 30 parágrafos expositivos para julgar a plausabilidade da sentença final, ou seja, a inferência que deveria ser gerada durante a leitura da $3^{3}$ ou $4^{-a}$ sentença. Por exemplo, no contexto: "O álcool e os antibióticos não são uma boa combinação. Aparentemente, o álcool diminui os efeitos da medicação. As pessoas demoram mais tempo para se recuperar quando elas bebem durante o tratamento.", a inferência esperada é a de que: "O álcool deveria ser evitado quando as pessoas tomam antibiótico". Todos os parágrafos apresentavam 4 sentenças e cada parágrafo tinha 2 versões: uma congruente com a sentença final e uma incongruente (ou seja, as sentenças intermediárias do contexto acima eram: "Os médicos recomendam tomar iogurte enquanto tomamos antibiótico. Alguns antibióticos destroem a flora intestinal.). Ao final da leitura de cada parágrafo, o participante tinha que selecionar a tecla 1 para: "Concordo que a última sentença é plausível com a conclusão do parágrafo" ou a tecla 2 , "Não concordo". Os parágrafos foram apresentados palavra por palavra, centralizada na tela do computador, em letras pretas, fonte Arial. Foi feita uma pausa de 3 a 5 minutos, ao final da leitura do trigésimo parágrafo (para detalhamento do método de pesquisa, ver BARETTA et al., 2009).

\footnotetext{
${ }^{11}$ Como a pesquisa foi conduzida na Universidade de Auckland, Nova Zelândia, no Centro de Neurociência Cognitiva, Departamento de Psicologia, o projeto foi aprovado por aquele Comitê de Ética em Pesquisa com Seres Humanos, sob número 2007/104.

12 Termo de Consentimento Livre e Esclarecido.
}

Dentre os vários resultados obtidos com o estudo, nos deteremos à discussão dos três principais. Primeiro, a análise do N400 mostrou que os participantes da pesquisa apresentaram maior dificuldade para processar o texto expositivo, conforme ápice do N400 na sentença 4 (onde a inferência referente ao parágrafo era explicitamente apresentada) e na sentença 3 (onde a informação complementar era dada para evocar a inferência da próxima sentença). Ou seja, os parágrafos expositivos mostraram maior latência no N400 quando comparados com os parágrafos narrativos, sinalizando, dessa forma, falhas do leitor para organizar as informações sendo processadas. Este resultado está em concordância com a literatura sobre compreensão do discurso, que argumenta que as narrativas são mais "fáceis" de se compreender que outros tipos de texto (NOORDMANN et al., 1992; TRABASSO; MAGLIANO, 1996). Segundo, a análise do componente N1 (baixas amplitudes) revelou que o hemisfério direito não faz distinção entre palavras de função (verbos auxiliares, artigos complementos, conjunções, conectivos, proposições, pronomes) e conteúdo (substantivos, verbos, a maioria dos adjetivos e advérbios com sufixação $-l y$, em inglês), demonstrando um processamento semântico mais global. O hemisfério esquerdo, por outro lado, demonstrou certa tendência ao priorizar por uma classe de palavra para processar (de função ou de conteúdo), revelando uma computação mais detalhada, fine-grained, conforme proposto por JungBeeman (2005). Terceiro, com relação ao envolvimento de diferentes áreas do hemisfério direito e hemisfério esquerdo no processo de geração de inferências, não houve diferenças significativas com relação ao tipo de texto. No entanto, o fato de que foi demonstrado que há envolvimento global de ambos os hemisférios cerebrais no processo de inferência e que o hemisfério direito é o mais participativo do processo, especialmente quando os participantes processavam a última sentença do parágrafo, está de acordo com estudos anteriores (MASON; JUST, 2004; SCHMALHOFFER et al., 2005; VIRTUE et al., 2005) e é congruente com o sistema de processamento semântico bilateral múltiplo, proposto por Jung-Beeman (2005). Finalmente, apesar de os componentes N400 e N1 são se mostrarem tão proeminentes quanto em estudos anteriores, principalmente como no estudo de St. George (1995), os resultados sugerem que o tipo de texto interfere na maneira que o cérebro processa e organiza a informação, principalmente nos níveis mais elevados de compreensão, como o processo de inferência.

\section{Considerações finais}

Considerando as pesquisas apresentadas, percebemos algumas das necessidades e possibilidades dos estudos na 
área da leitura e a importância de se conhecerem diferentes métodos de investigação dos processos envolvidos.

As interfaces provenientes de diferentes áreas do conhecimento têm possibilitado tanto o desenvolvimento de novos métodos quanto o refinamento de métodos investigativos já existentes para atender às necessidades de pesquisas com menor custo para o pesquisador. No caso das pesquisas relatadas neste artigo, é importante relembrar que tanto o Translog quanto o EEG foram desenvolvidos, inicialmente, para a pesquisa de outras facetas cognitivas: o primeiro para investigar os processos inerentes à escrita em tradução e o segundo para o estudo clínico e diagnóstico de epilepsia e distúrbios do sono. Há que se destacar, que no caso do EGG, apesar do custo de uma sessão ser relativamente inferior ao custo de uma sessão com o fMRI, por exemplo, o preparo do participante requer um procedimento relativamente demorado (de 10 a 15 minutos) ao colocar a touca e aplicar a solução condutora dos sinais elétricos em cada eletrodo. Esses 15 minutos, dependendo da tarefa proposta, são valiosos ao se recrutarem participantes que normalmente não dispõem de muito tempo.

Um dos benefícios de ambas as tecnologias apresentadas relacionam-se ao fato de elas apresentarem risco baixo aos participantes e de oferecem grandes benefícios quanto aos resultados que podem vislumbrar, devido à qualidade e precisão dos dados cognitivos originados durante a coleta, abrindo novas e diferentes janelas de possibilidades de pesquisa. Em ambos os estudos descritos neste artigo, pode-se perceber que o fator tempo é uma variável a ser considerada nas pesquisas que envolvem o processamento em leitura, pois a rapidez com que um texto é lido pode levar a uma falsa interpretação de compreensão bem sucedida. Outro benefício que é digno de ser reforçado é o acesso e custo menor do que as demais tecnologias mencionadas e comumente utilizadas para a investigação em leitura (fMRI, PET scan, MEG), normalmente restrita a grandes centros de pesquisa. $\mathrm{O}$ Translog em especial pode ser utilizado com grupos de participantes com número mais expressivo, considerando, em parte, os custos e a viabilidade de se ter um computador em diferentes lugares, da mata à metrópole. Ressaltamos, no entanto, que o acesso aos dados, diante dos métodos abordados, serão sempre indiretos e a engenhosidade dos métodos e técnicas requerem cuidado e cautela, bem como a necessidade de reduplicação dos estudos a fim de aumentar a segurança e a confiabilidade na interpretação dos resultados.

\section{Referências}

ALVES, Fabio Alves. Triangulação como opção metodológica em pesquisas empírico-experimentais em tradução. In:
PAGANO, A. (Org.). Metodologia de pesquisa em tradução. 2001. p. 69-92. (Estudos linguísticos, 3).

ASCHCRAFT, Mark H. Human memory and cognition. New York: Harper Collins, 1994.

BARETTA, Luciane; TOMITCH, Lêda Maria Braga; MACNAIR, Nicolas; LIM, Vanessa Kwan; WALDIE, Karen Elizabeth. Inference making while reading narrative and expository texts: An ERP study. Psychology \& Neuroscience, v. 2, n. 2, p. 3-9, dez. 2009.

BERBER SARDINHA, Tony (Org.). A língua portuguesa no computador. São Paulo: Mercado de Letras, 2005.

BROWN, Colin; HAGGORT, Peter. On the electrophysiology of language comprehension: Implications for the human language system. In: CROCKER, Matthew W.; PICKERING, Martin; CLIFTON JR. Charles. (Org.). Architectures and mechanisms for language processing. Cambridge University Press, 2000. p. 213-237.

BUCHWEITZ, Augusto; LIMBERGER, Bernardo Kolling; KRAMER, Rossana (Org.). Letrônica - Revista digital do PPGL, PUCRS, v. 7, n. 1, ago. 2014.

COULSON, Seana; VAN PETTEN, Cyma. Conceptual integration and metaphor: An event-related potential study. Memory and Cognition, v. 30, n. 6, p. 958-968, 2002. http:// dx.doi.org/10.3758/BF03195780

DEHAENE, Stanislas. Os neurônios da leitura: como a ciência explica a nossa capacidade de ler. Tradução de Leonor ScliarCabral. Porto Alegre: Penso, 2012.

FINGER-KRATOCHVIL, Claudia. Estratégias para o desenvolvimento da competência lexical: relações com a compreensão em leitura. 2010. 677p. (v.) I, (v.) II. Tese (Doutorado em Linguística) - Universidade Federal de Santa Catarina, Florianópolis, 2010.

GROFF, Patrick. The usefulness of pseudowords. Disponível em: <http://www.nrrf.org/essay_pseudowords.htm>. Acesso em: 30 nov. 2008.

HAUSMANN, Markus; BECKER, Claudia; GATHER, Ursula; GÜNTÜRKÜN, Onur. Functional cerebral asymmetries during the menstrual cycle: a cross-sectional and longitudinal analysis. Neuropsychologia, v. 40, n. 7, p. 808-816, 2002. http://dx.doi. org/10.1016/S0028-3932(01)00179-8

JUNG-BEEMAN, Mark. Bilateral brain processes for comprehending natural language. TRENDS in Cognitive Sciences, v. 9, n. 11, p. 512-518, 2005. http://dx.doi. org/10.1016/j.tics.2005.09.009

KUTAS, Marta; VAN PETTEN, Cyma. Psycholinguistics electrified: event-related brain potential investigations. In: GERNASBACHER, Morton Ann (Org.). Handbook of psycholinguistics. San Diego, CA: Academic Press. 1994. p. 83-143.

KUTAS, Marta; Hillyard, Steven A. Reading senseless sentences: Brain potentials reflect semantic incongruity. Science, v. 207, p. 203-205, 1980. http://dx.doi.org/10.1126/ science. 7350657

KUTAS, Marta; SCHMITT, B. M. Language in microvolts. In: BANICH, M.T.; MACK, M. (Ed.). Mind, brain and languagemultidisciplinary perspectives. Mahwah, New Jersey: Lawrence Erlbaum Associates, 2003. p. 171-209. 
LAGE, Aleria Cavalcante. Análise de novos dados linguísticos: a eletroencefalografia em neurociência da linguagem. Revista $F S A$, v. 10, p. 153-172. jan.-mar. 2013. Disponível em: $<$ http://dx.doi.org/10.12819/2013.10.1.9>. http://dx.doi. org/10.12819/2013.10.1.9

MASON, Robert; JUST, Marcel, Adam. How the brain processes causal inferences in text: A multiple process theory of language function in both hemispheres. Psychological Science, v. 15, p. 1-7, 2004. http://dx.doi.org/10.1111/j.09637214.2004.01501001.x

NATION; Kate; COCKSEY, Joanne. The relationship between knowing a word and reading it aloud in children's word reading development. Journal of experimental child psychology, v. 103, p. 296-308, 2009. http://dx.doi.org/10.1016/j.jecp.2009.03.004

NATION, Kate; SNOWLING, Margaret. Semantic processing and development of word recognition skills: evidence from children with reading comprehension difficulties. Journal of memory and language, v. 39, p. 85-101, 1998. http://dx.doi. org/10.1006/jmla.1998.2564

NOORDMANN, Leo.G.M., VONK, Wietske. Reader's knowledge and the control of inferences in reading. Language and Cognitive Processes, v. 7, n. 3/4, p. 373-391, dez.1992.

NY, Jean-François Le; CORDIER, Françoise. Contribution of word meaning and components of familiarity to lexical decision: a study with pseudowords constructed from words with known or unknown meaning. Current psychology letters, V. 1, n. 12, 2004.

PERFETTI, Charles. Reading ability: lexical quality to comprehension. Scientific Studies of Reading, v. 11, n. 4, p. 357-383, 2007.http://dx.doi.org/10.1080/10888430701530730

RANEY, GE. Monitoring changes in cognitive load during reading: An event-related brain potential and reaction time analysis. Journal of Experimental Psychology: Learning, Memory and Cognition, v. 19, n. 1, p. 51-69, 1993. http://dx.doi. org/10.1037/0278-7393.19.1.51

RUDDELL, Martha R. Vocabulary knowledge and comprehension: a comprehension-process view of complex literacy relations. In: RUDDELL, Robert B.; RUDDELL, Martha R.; SINGER, Harry. Theoretical models and processes of reading (fourth edition). Newark, DE: International Reading Association, 1994. p. 414-447.

SCHMALHOFFER, Franz; FRIESE, UWE; PIETRUSKA, KARIN; RAABE, Markus; RUTTSCHMANN, Roland. Brain Processes of Relating a Statement to a Previously Read Text:
Memory Resonance and Situational Constructions. Proceedings CogSci2005: XXVIII annual conference of the cognitive science society, Italy, v. 21-23, p. 1949-1954, jul. 2005.

SCLIAR-CABRAL, Leonor. À propos de la reconnaissance des unités lexicales. Linx, v. 29, p. 73-84, 1993. http://dx.doi. org/10.3406/linx.1993.1273

ST. GEORGE, Marianne. An investigation of the nature and time-course of inferences using event-related brain potentials. 1995. Tese (Doutorado) - University of Delaware, Newark, 1995.

TANNER, Jared. 6 julho 2007. Pet scans and fMRI compared. Disponível em: <http://www.brainybehavior.com/blog/ 2007/07/pet-scans-and-fmri-compared/>. Acesso em: 20 maio 2014.

TOMITCH, Lêda Maria Braga (Org.). The neuroscience of reading. Ilha do Desterro, v. 63, jul.-dez. 2012.

TOMITCH, Lêda Maria Braga; JUST, Marcel adam; NEWMAN, Sharlene. A neuroimagem funcional na investigação do processo de leitura. In: TOMITCH, Lêda Maria Braga; RODRIGUES, Cassio (Org.). Linguagem e cérebro humano: contribuições interdisciplinares. Porto Alegre: ARTMED, 2004. p. 167-176.

TRABASSO, Tom; MAGLIANO, Joseph P. Conscious understanding during comprehension. Discourse Processes, v. 21, p. 255-287, nov. 1996. http://dx.doi.org/10.1080/ 01638539609544959

OCDE - Organização de cooperação e desenvolvimento econômicos. Compreendendo o cérebro - rumo a uma nova ciência do aprendizado. Tradução de Eliana Rocha. São Paulo: Editora Senac, 2003.

VENEZKY, Richard. The American way of spelling: the structure and origins of American English orthography. New York: Guilford Press, 1999.

VIRTUE, Sandra; HABERMAN, Jason; CLANCY, Zoe; PARRISH, Todd; JUNG-BEEMAN, Mark. Neural activity of inferences during comprehension. Brain Research, n. 1084, p. 104-114, 2005.

WANNMACHER PEREIRA, Vera; GUARESI, Ronei (Org.). Estudos sobre leitura: psicolinguística e interfaces. EDIPUCRS, 2012.

Recebido: 31 de agosto de 2014

Aprovado: 07 de novembro de 2014

Contatos:barettaluciane@gmail.com cfkrato@gmail.com 